\title{
A multipurpose activity platform utilized in the open-field setting
}

\author{
H. P. PFISTER, R. R. MUDGE, and A. O. HARCOMBE \\ University of Newcastle, Newcastle, New South Wales, Australia
}

\begin{abstract}
An automated multipurpose activity platform (MAP) that can be used to measure activity in the horizontal plane is described. Detection of the position of a small-bodied animal is based on completion of an electronic circuit in one or several cells of a 21 by 21 matrix below the floor. The MAP is constructed on the basis of an 11 by 11 matrix, but, through electronic averaging, operates functionally on the basis of a 21 by 21 matrix. The MAP has no built-in data storage facility and needs to be interfaced with a recording system. The system has been successfully utilized in measuring open-field activity of the rats.
\end{abstract}

A fully automated multipurpose activity platform (MAP) was designed on the principle of a floor or platform contact, utilizing an electronic matrix. The main advantage of the MAP is that it can be adapted to measure horizontal activity in a number of different settings (e.g., T-maze, Y-maze, Hebb-Williams maze, runway, open-field, etc.). In general, activity is defined in terms of change of location of the subject on the MAP over time. The unit is designed such that, at any time, the location of the animal is given in coordinates based on the underlying matrix. As the MAP has no facility for recording or storing coordinate data, interfacing with a recording system (e.g., digital voltmeter, two-channel recorder, polygraph, computer, etc.) is necessary.

To date, the MAP has been successfully utilized with rats in an open-field setting. A circular-shaped frame, $96 \mathrm{~cm}$ in diam and $30 \mathrm{~cm}$ high is suspended $2 \mathrm{~mm}$ above the surface of the MAP. For the setting described here, the MAP is interfaced through an A/D converter with a PDP-12 computer, permitting the storage of coordinates on Linc tape.

The use of the MAP in the open-field setting offers certain advantages over other automated systems. For example, the automated open field designed by Morgret and Albee (1974) does not record true diagonal movements of the animal across the platform, and data is lost. The open field designed by Denenberg, Gartner, and Myers (1975) is based on a center-ofpressure force platform which is connected through four-corner mounted electromechanical force transducers onto the main frame.

To obtain the coordinates of an animal in the open field, the values generated by the four transducers, as well as the subject's body weight, must be considered. The Denenberg et al. (1975) open field also must be calibrated before it can be used. This calibration is unnecessary in either the Morgret and Albee (1974) or the present design. Because of the principle of force employed in the Denenberg et al. (1975) design, foreign material (e.g., fecal boli) can distort the true balance of the four transducers and thus erroneous coordinates can be generated. The MAP eliminates such problems.

\section{APPARATUS}

The MAP was designed as an 11 by 11 cell matrix, each cell measuring $8.5 \times 8.5 \mathrm{~cm}$. It consists of a base plate (see Figure 1) made of 11 strips of copper-clad laminate (A) measuring $8.5 \times 8.5 \mathrm{~cm}$, spaced $.3 \mathrm{~cm}$ apart and glued to a 2-cm-thick wooden base (B) measuring $96.5 \times 96.5 \mathrm{~cm}$. The face of the 11 strips of copper-clad laminate forms the $\mathrm{Y}$ axis of the MAP. To prevent the corrosion of the copper-clad laminate, all copper strips must be sprayed with silvering solution. A suspended wire grid (C), consisting of No. 30 standard wire gauge Nichrome spring wire is suspended above the base plate. The grid is organized into 11 units, each containing 14 wires approximately $.6 \mathrm{~cm}$ apart. The uniform suspension of the wire grid is maintained by pretensioning of each individual wire. The wire grid is supported by strips of $.3-\mathrm{cm}$-wide nonconductive tape. These tapes are located in the gap (see Figure 1) between the copper-clad laminates and protrude $.02 \mathrm{~cm}$ above the copper surface. To insure good cable connections with the Nichrome wire (see Figure 2), a phosphoric acid-based soldering flux should be used. As shown in Figure 1, the wire grid is covered by one piece of .1-cm-thick soft PVC vinyl.

The 11 copper laminate strips (A) are supplied with 1 to $11 \mathrm{~V}$ at $1-\mathrm{V}$ intervals. As a result, the first copper strip has a voltage of 1 and the last has a voltage of 11. To make contact between one of the 11 wire grids and one of the copper-clad laminates (i.e., to close the circuit), requires a minimum weight of $30 \mathrm{gm} / \mathrm{cm}^{2}$. The resulting voltage level originating from the copper-clad laminate gives the $\mathrm{Y}$ coordinate; the position of the wire grid making contact produces the $\mathrm{X}$ coordinate. 


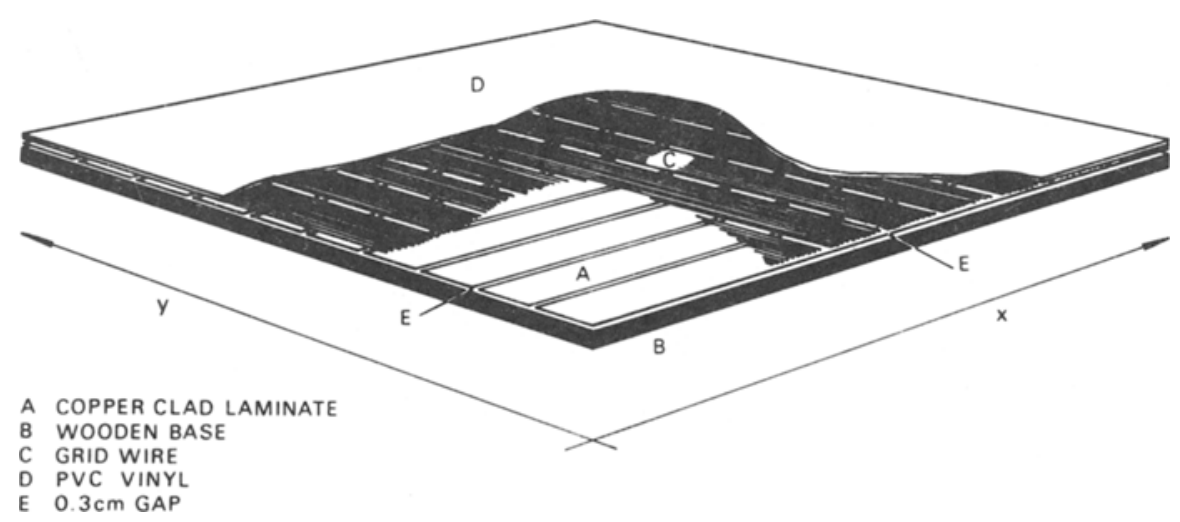

Figure 1. The multipurpose activity platform (MAP).

There is a maximum of 11 different voltage inputs on the $\mathrm{Y}$ axis through the 11 copper strips. A maximum of 11 different output positions for these voltage levels is produced on the $\mathrm{X}$ axis through the wire grid.

The 11 copper-clad laminate (Y-axis) inputs are connected to 11 adjustable emitter followers that are tied together (see the circuit diagram in Figure 2). Such an arrangement allows for one $Y$ output only by summation of any number of inputs on the $Y$ axis over the number of input. If an animal makes contact over two copper-clad laminates, the two voltage levels are reduced to one by summation over the number of inputs, creating a new coordinate. This allows for the 11 coordinates in the $\mathrm{Y}$ plane to be expanded electronically to a total of 21 .

The output signal obtained on the wire grid (X plane) is fed to a series of LM 339 comparators that are activated as soon as a voltage input is received. These comparators are connected to MC 14016 analog switches that allow for the summation of any number of voltage levels received through the wire grid (X plane). This arrangement allows all signals of the $X$ plane to be averaged; e.g., two voltage levels received on two adjacent wire grids are averaged and a new coordinate results. Such a design expands electronically the 11 $\mathrm{X}$ coordinates to a total of 21 .

Thus, the MAP functionally consists of 21 by 21 cells, each measuring $4.25 \times 4.25 \mathrm{~cm}$, but at this stage the $\mathrm{X}$ and $\mathrm{Y}$ output of the MAP must be connected to a suitable recording system.

The MAP is simple to construct, relatively inexpensive, and, once set up, maintenance free. Finally, the system can be utilized in a variety of behavioral settings by simple manipulation of dividers or walls above the platform.

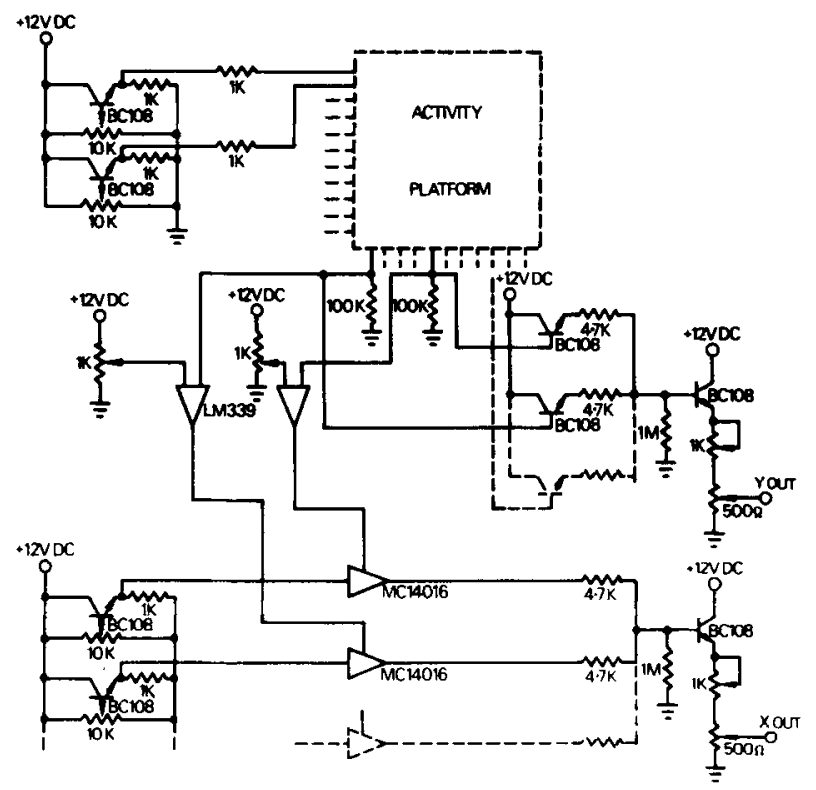

Figure 2. Schematic of electronic circuit.

\section{REFERENCES}

Denenberg, V. H., Gartner, T., \& MYers, M. Absolute measurement of open-field activity in mice. Physiology and Behavior, 1975, 15, 505-509.

Morgret, M. K., \& Albee, P. R. An automated open-field apparatus utilizing an improved resistance detection circuit. Behavior Research Methods \& Instrumentation, 1974, 6, 327-328.

(Received for publication June 24, 1977; revision accepted September 20, 1977.) 